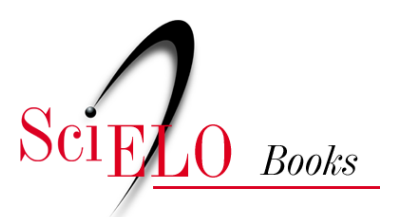

\title{
16 Tribo Senecioneae Cass.
}

\author{
Aristônio M. Teles \\ Fernanda Santos Freitas
}

TELES, A., and FREITAS, F.S. Tribo Senecioneae Cass. In: ROQUE, N. TELES, A.M., and NAKAJIMA, J.N., comp. A família Asteraceae no Brasil: classificação e diversidade [online]. Salvador: EDUFBA, 2017, pp. 123-130. ISBN: 978-85-232-1999-4. https://doi.org/10.7476/9788523219994.0018.

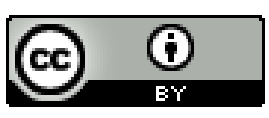

All the contents of this work, except where otherwise noted, is licensed under a Creative Commons Attribution 4.0 International license.

Todo o conteúdo deste trabalho, exceto quando houver ressalva, é publicado sob a licença Creative Commons Atribição $\underline{4.0}$. 


\title{
TRIBO SENECIONEAE CASS.
}

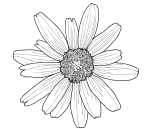 \\ Aristônio M. Teles \\ Fernanda Santos Freitas
}

Senecioneae Cass. é a maior tribo de Asteraceae em número de espécies com aproximadamente 3 mil espécies e cerca de 150 gêneros (NORDENSTAM et al., 2009). A tribo foi tradicionalmente dividida nas subtribos Blennospermatinae, Senecioninae e Tussilagininae (BREMER, 1994). No entanto, análises filogenéticas recentes baseadas em informações moleculares sugerem que a tribo seja dividida em 4 subtribos: Abrotanellinae, Othonninae, Senecioninae e Tussilagininae (NORDENSTAM et al., 2009; PELSER et al. 2007). Dessas subtribos, apenas Senecioninae possui espécies ocorrentes no Brasil.

Apesar da grande riqueza em número de espécies da tribo, a maioria dos táxons pode ser reconhecida primariamente pelas brácteas involucrais, que são de tamanhos iguais entre si e que estão dispostas em uma única série (BREMER, 1994; PELSER et al., 2007). Frequentemente, esse invólucro unisseriado possui uma série externa de brácteas que é conhecida como calículo (BREMER, 1994). Por outro lado, a tribo apresenta uma grande variação morfológica em outras características, como formato e tamanho da lâmina foliar, indumento, capitulescência, tipo de capítulo e coloração das flores (NORDENSTAM et al., 2009).

Além de possuir características morfológicas singulares, as espécies de Senecioneae são caracterizadas por acumularem compostos químicos secundários, como alcaloides pirrolizidínicos e sesquiterpenos do tipo furanoeremofilanos (ROBINS, 1977). Devido à presença desses alcaloides, muitas espécies são altamente hepatotóxicas, particularmente ao gado. 
Senecioneae possui distribuição cosmopolita (BREMER, 1994); no entanto, há importantes centros geográficos de especiação e diversificação de gêneros. No continente africano, a maior diversidade de gêneros é encontrada na África do Sul (25 gêneros), enquanto que no continente americano, as maiores diversidades são encontradas no México (18 gêneros), Peru (17 gêneros) e Colômbia (15 gêneros) (NORDENSTAM et al., 2009). As espécies da tribo ocorrem predominantemente em áreas temperadas e subtropicais áridas ou regiões montanhosas (NORDENSTAM, 2007).

\section{Descrição}

Ervas, arbustos, lianas ou árvores. Folhas alternas, em roseta ou distribuídas ao longo dos ramos, sésseis ou pecioladas, inteiras ou pinatissectas. Capitulescência corimbiforme, paniculiforme ou tirsoide, terminal ou axilar ou capítulo solitário. Capítulos radiados, disciformes ou discoides, heterógamos ou homógamos; invólucro unisseriado, caliculado ou ecaliculado; brácteas involucrais livres ou conatas; receptáculo plano, convexo ou cônico, desprovido de projeções ou fimbriado a denticulado, piloso ou glabro, meduloso ou fistuloso. Flores do raio pistiladas, corola liguliforme ou tubuloso-filiforme, flores do disco bissexuadas, corola tubulosa 5-lobada, corola geralmente amarela, algumas vezes branca, alaranjada, rosada, vermelha ou lilás; estames com anteras apendiculadas apicalmente, base obtusa, sagitada ou caudada; tecido endotecial com células com espessamento radial ou polarizado, raramente transicional; colar da antera reto, uniforme ou basalmente dilatado (balustriforme) com longas células; estilete bífido, apicalmente truncado, obtuso, arredondado ou triangular, algumas vezes longamente acuminado com tricomas fusionados ou com distinto tufo de tricomas; áreas estigmáticas contínuas ou separadas. Cipsela costada, cilíndrica ou obovoide, glabra ou variavelmente pubescente; pápus de poucas a muitas cerdas, persistentes ou caducas, uni a multisseriadas, finas, brancas, estramíneas ou púrpura.

No Brasil, Senecioneae está representada por 8 gêneros e aproximadamente 100 espécies, concentradas especialmente nos campos rupestres e campos de altitude das serras e montanhas das regiões Sudeste e Sul. 
Algumas espécies exóticas são utilizadas como ornamentais, a exemplo de Curio rowleyanus (H. Jacobsen) P.V. Heath, Senecio flaccidus Less., S. viravira Hieron. e Senecio tamoides DC. Outras, como Pseudogynoxys cabrerae H. Rob. \& Cuatrec., Emilia fosbergii Nicolson (Figura 14E) e E. sonchifolia (L.) DC., ocorrem como subespontâneas. Representantes de Senecioneae são encontrados na Figura 14D-I.

\section{Chave de identificação para os gêneros de Senecioneae no Brasil}

1. Capítulos discoides; brácteas involucrais e flores do capítulo em número de 5 Hoehnephytum

1'. Capítulos disciformes, radiados ou discoides; porém, se discoides, com brácteas involucrais e flores do capítulo em número maior que 5 2

2. Capítulos disciformes, flores periféricas com corola longamente filiforme (Figura 9I) Erechtites

2'. Capítulos radiados, flores do raio com corola distintamente liguliforme, e/ou capítulos discoides, com todas as flores com corola tubulosa 3

3. Ramos do estilete com ápice convexo (Figuras 9G,J) ou truncado (Figuras 9K-L)

3'. Ramos do estilete com ápice truncado, com conspícuo tufo de tricomas partindo do centro (Figura 9J), ou apendiculado, longamente acuminado, com tricomas fusionados (Figura 13A) ou apêndice penicilado 7

4. Capítulos discoides Senecio

4'. Capítulos radiados 5

5. Ramos do estilete com ápice convexo (Figura 9G) Dendrophorbium

5'. Ramos do estilete com ápice truncado (Figuras 9K-L) 6

6. Plantas escandentes. Pentacalia

6'. Plantas eretas, prostradas ou decumbentes Senecio 
7. Ramos do estilete com ápice truncado, com conspícuo tufo de tricomas partindo do centro, circundado por coroa de tricomas curtos, divergentes (Figura 9J) Graphistylis

7'. Ramos do estilete com ápice apendiculado, longamente acuminado, com tricomas fusionados (Figura 13A) ou apêndice penicilado, sem coroa de tricomas divergentes 8

8. Subarbustos a arbustos escandentes, perenes; capítulos radiados; invólucro caliculado; ramos do estilete com ápice longamente acuminado, com tricomas fusionados (Figura 13A) Pseudogynoxys 8'. Ervas eretas, anuais; capítulos discoides; invólucro ecaliculado (Figura 9H); ramos do estilete com ápice com apêndice penicilado Emilia

\section{Literatura recomendada}

BAKER, J. G. Compositae IV: Helianthoideae - Mutisiaceae. In: MARTIUS, C. F. P.; EICHLER, A. G., Flora Brasiliensis. Monachii: Lipsiae, 1884. v. 6, pt. 3, p. 135-398.

BELCHER, R. O. A revision of the genus Erechtites (Compositae), with inquiries into Senecio and Arrhenechthites. Annals of the Missouri Botanical Garden, Saint Louis, v. 43, n. 1, p. 1-85, 1956.

BREMER, K. Asteraceae: cladistics and classification. Portland: Timber Press, 1994.

CABRERA, A. L. Notes on the Brazilian Senecioneae. Brittonia, [Bronx], v. 7, n. 2, p. 53-74, 1950.

CABRERA, A. L. El género Senecio (Compositae) en Brasil, Paraguay y Uruguay. Arquivos do Jardim Botânico do Rio de Janeiro, Rio de Janeiro, v. 15, p. 163-264, 1957.

CABRERA, A. L.; KLEIN, R. M. Compostas - Tribo: Senecioneae. In: REITZ, R. Flora ilustrada catarinense. Itajaí: Herbário Barbosa Rodrigues, 1975. p. 126-222. 
JEFFREY, C. Notes on Compositae, VI: The tribe Senecioneae (Compositae) in the Mascarene Islands with an annotated world check-list of the genera of the tribe. Kew Bulletin, London, v. 47, p. 49-109, 1992.

HIND, D. J. N. Pseudogynoxys cabrerae; Compositae. Curtis's Botanical Magazine, [Oxford], v. 9, n. 4, p. 153-200, 1992.

HIND, D. J. N. A checklist of the Brazilian Senecioneae (Compositae). Kew Bulletin London, v. 48, n. 2, p. 279-295, 1993.

HIND, D. J. N. The tribe Senecioneae (Compositae) in Bahia, Brazil, with descriptions of a new section and species in Senecio. Kew Bulletin, London, v. 54 , n. 4 , p. $897-904,1999$.

JEFFREY, C. The tribe Senecioneae (Compositae) in the Mascarene Islands with an annotated world check-list of the genera of the tribe: Notes on Compositae: VI. Kew Bulletin, London, v. 47, n. 1 p. 49-109, 1992.

MATZENBACHER, N. I. O complexo "Senecionóide" (Asteraceae - Senecioneae) no Rio Grande do Sul - Brasil. Porto Alegre: Instituto de Biociências, Universidade Federal do Rio Grande do Sul, Tese de Doutorado. 1998. $274 \mathrm{p}$.

NORDENSTAM, B. Taxonomic studies in the tribe Senecioneae (Compositae). Lund: Lund Botanical Society, 1978. p. 1-83. (Opera Botanica, v. 44)

NORDENSTAM, B. XII. The tribe Senecioneae Cass. 1819. In: KADEREIT, J. W.; JEFFREY, C. (Ed.). The Families and Genera of Vascular Plant: v. VIII:

Flowering Plants Eudicots: Asterales. Berlin; Springer, 2007. p. 208-241.

NORDENSTAM, B. et al. Senecioneae. In: FUNK, V. A. et al. (Ed.). Systematics, evolution and biogeography of Compositae. Vienna: IAPT, 2009. p. 503-525.

PELSER, P. B. et al. An ITS phylogeny of tribe Senecioneae (Asteraceae) and a new delimitation of Senecio L. Taxon, Utrecht v. 56, n. 4, p. 1077-1104, 2007. 
PRUSKI, J. F. Pseudogynoxys lobata (Compositae: Senecioneae), a new species from Bolivia and Brazil. Systematic Botany, Kent, v. 21, n. 1, p. 101-105, 1996.

ROBINS, D. J. Senecioneae - chemical review. In: HEYWOOD, V. H.;

HARBORNE, J. B.; TURNER, B. L. The biology and chemistry of the Compositae. London: Academic Press, 1977. p. 831-850.

ROBINSON, H.; CUATRECASAS, J. Notes on the genus and species limits of Pseudogynoxys (Greenm.) Cabrera (Senecioneae, Asteraceae). Phytologia,Huntsville, v. 36, p. 177-192, 1977.

ROBINSON, H.; CUATRECASAS, J. A review of the Central American species of Pentacalia (Asteraceae: Senecioneae). Phytologia, Huntsville, v. 40, p. $37-50,1978$.

TELES, A. M. Nova combinação e chave revisada para Dendrophorbium (Asteraceae - Senecioneae) no Brasil. Rodriguésia, Rio de Janeiro, v. 61, n. 1, p. 143-145, 2010.

TELES, A. M.; FREITAS, F. S. Senecio hortensiae (Asteraceae, Senecioneae): a new species from Espírito Santo, Brazil. Phytotaxa, Auckland, v. 142, n. 1, p. 46-50, 2013.

TELES, A. M.; NAKAJIMA, J. N.; STEHMANN, J. R. Senecio albus, a new species of Senecio sect. Adamantina (Senecioneae - Asteraceae) with an emendment to the section. Kew Bulletin, London, v. 64, n. 1, p. 161-165, 2009.

TELES, A. M.; STEHMANN, J.R. 2011. Flora da Serra do Cipó, Minas Gerais: Asteraceae - Senecioneae. Boletim de Botânica da Universidade de São Paulo, São Paulo, v. 29, n. 1, p. 57-68, 2011.

TELES, A. M.; STEHMANN, J. R. 2016. A tribo Senecioneae (Asteraceae) em Minas Gerais, Brasil. Rodriguésia, Rio de Janeiro, v. 67, n. 2, p. 455-487, 2016. 


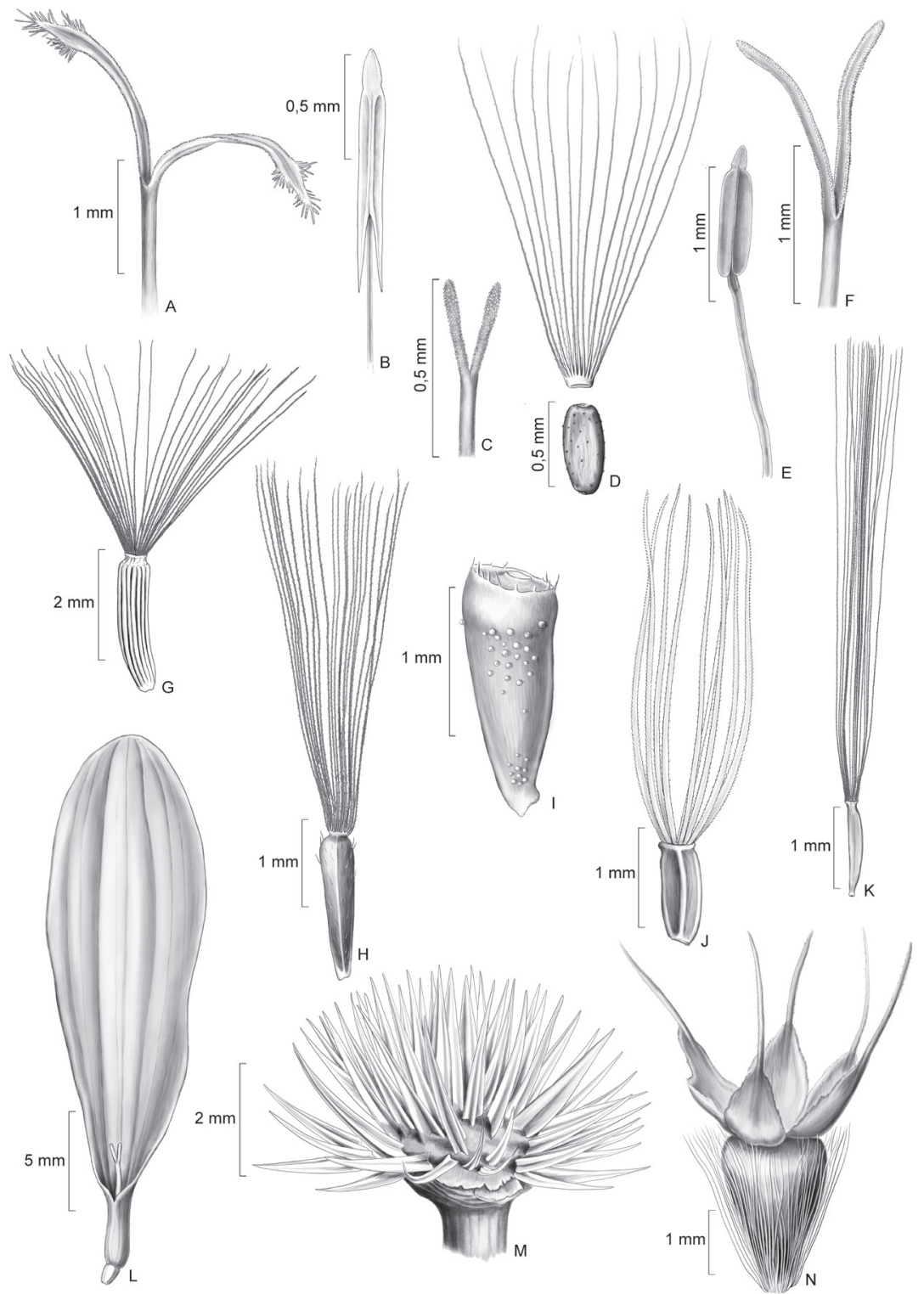

Figura 13. Senecioneae. A. Estilete de Pseudogynoxys cabrerae H.Rob. \& Cuatrec. Gnaphalieae. B-C. Chionolaena jeffreyi H. Rob.: B. Antera; C. Ramos do estilete. D. Cipsela e pápus de Gamochaeta pensylvanica (Willd.) Cabrera. Astereae. E-G. Baccharis aleluia A.S. Oliveira \& Deble: E. Antera ecaudada; F. Ramos do estilete; G. Cipsela e pápus; H. Cipsela e pápus de Conyza primulifolia (Lam.) Cuatrec. \& Lourteig; I. Cipsela e pápus de Egletes viscosa (L.) Less.; J. Cipsela e pápus de Grindelia brachystephana Griseb.; K. Cipsela e pápus de Podocoma notobellidiastrum (Griseb.) G.L. Nesom. Anthemideae. L. Flor ligulada feminina de Chrysanthemum sp. Helenieae. M-N. Gaillardia pulchella Foug.: M. Receptáculo paleáceo; N. Cipsela e pápus. 

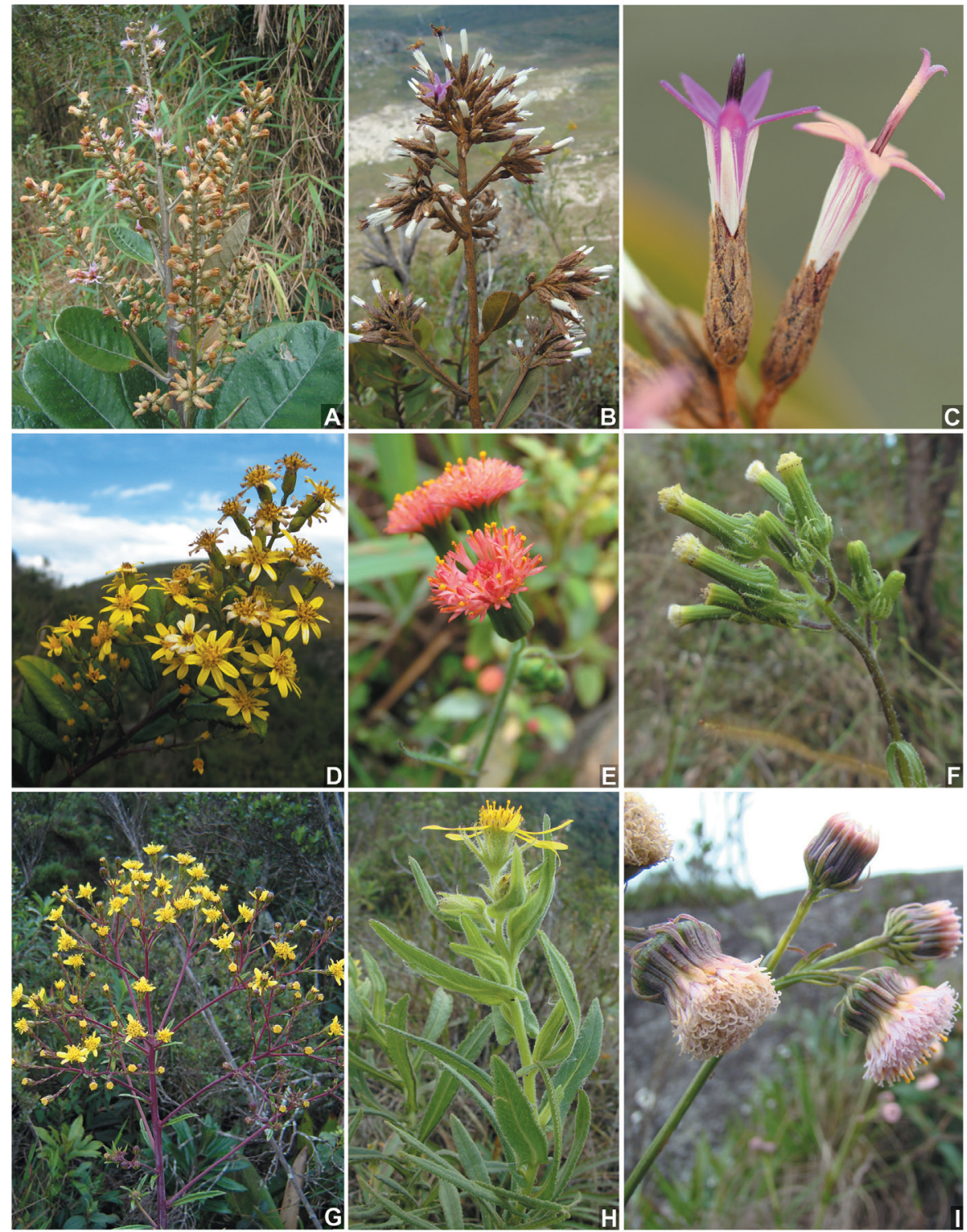

Figura 14. Moquinieae. A. Moquinia racemosa (Spreng.) DC.; B-C. Pseudostifftia kingii H. Rob. Senecioneae. D. Dendrophorbium pellucidinerve (Sch.Bip. ex Baker) C.Jeffrey; E. Emilia fosbergii Nicolson; F. Erechtites hieracifolius (L.) Raf. ex DC.; G. Graphistylis dichroa (Bong.) D.J.N. Hind; H. Senecio oleosus Vell.; I. Senecio pohlii Sch. Bip. ex Baker. 